\title{
Integrable Renormalization I: the Ladder Case
}

\author{
KURUSCH EBRAHIMI-FARD* \\ I.H.É.S. \\ Le Bois-Marie, 35, Route de Chartres \\ F-91440 Bures-sur-Yvette, France \\ and \\ Universität Bonn - Physikalisches Institut \\ Nussallee 12, D-53115 Bonn, Germany \\ $\mathrm{LI} \mathrm{GUO}^{\dagger}$ \\ Rutgers University \\ Department of Mathematics and Computer Science \\ Newark, NJ 07102, USA \\ DIRK KREIMER ${ }^{\ddagger}$ \\ CNRS-I.H.É.S. \\ Le Bois-Marie, 35, Route de Chartres \\ F-91440 Bures-sur-Yvette, France
}

October 30, 2018

\begin{abstract}
In recent years a Hopf algebraic structure underlying the process of renormalization in quantum field theory was found. It led to a Birkhoff factorization for (regularized) Hopf algebra characters, i.e. for Feynman rules. In this work we would like to show that this Birkhoff factorization finds its natural formulation in terms of a classical r-matrix, coming from a Rota-Baxter structure underlying the target space of the regularized Hopf algebra characters. Working in the rooted tree Hopf algebra, the simple case of the Hopf subalgebra of ladder trees is treated in detail. The extension to the general case, i.e. the full Hopf algebra of rooted trees or Feynman graphs is briefly outlined.
\end{abstract}

*kurusch@ihes.fr

†liguo@newark.rutgers.edu

${ }^{\ddagger}$ kreimer@ihes.fr and dkreimer@bu.edu. Center for Math. Phys., Boston University. 


\section{Introduction}

In 1960 the mathematician Glen Baxter [1] used a simple algebraic identity to solve an analytic problem. Later, in 1963 F. V. Atkinson [2] gave a characterization of this relation in terms of a so-called subdirect Birkhoff decomposition. It was G.-C. Rota who investigated this identity more thoroughly and realized its importance within combinatorics and other fields in mathematics [3, 4, 5]. This identity is called the Rota-Baxter relation and will be introduced in the next section. See [6, 7] for reviews and [8, 9, 10, 11, 12, for recent developments relating this identity to other fields in mathematics.

The same relation in its Lie algebraic version was later rediscovered under the name (operator form of the modified) classical Yang-Baxter ${ }^{1}$ equation within the field of integrable systems [13, 14, 15]. See [16] for a nice review especially with respect to double Lie algebras and factorization theorems, i.e. the Riemann Hilbert problem.

Very recently the Rota-Baxter relation was found to be of crucial importance within the Hopf algebraic approach of Kreimer, and Connes and Kreimer to renormalization theory of perturbative quantum field theory [17, 18, 19, 20]. It implies a Birkhoff decomposition of Hopf algebra characters with the target space being a Rota-Baxter algebra. See [21] for a first introduction.

Our work aims at a clarification of the link between the last two subjects. We show how the Rota-Baxter relation on the target space of the Hopf algebra characters can be lifted to the Lie algebra of infinitesimal characters. Atkinson's theorem then implies an infinitesimal factorization on this space. This factorization in turn implies the Birkhoff decomposition for the Hopf algebra characters. We derive its twisted antipode form first found by one of us [17. There it was introduced in a formal way mainly motivated by its ability to reproduce Zimmermann's forest formula. Dimensional regularization together with a minimal subtraction scheme furnishes one special case (distinguished by its convenience in applied particle physics) of our approach in which the target space consists of the algebra of Laurent series with finite pole part. Its Rota-Baxter structure stems from the projection onto the pole part of a Laurent series. Here, we will discuss the algebraic Birkhoff decomposition for general Rota-Baxter maps $R$, provided by various choices of renormalization schemes. Let us mention here that we will restrict our attention later on to the case of the Hopf subalgebra of rooted ladder trees. In this manner we can avoid lengthy formulas generated by the Baker-CampbellHausdorff formula, and can treat this case in full technical detail. In the end it will be outlined though how the general case, i.e. for arbitrary rooted trees or graphs, is derived. Details and also a mathematically more rigorous presentation will be given in a forthcoming paper, emphasizing the link to integrable systems.

The paper is structured as follows. The next section introduces the notion of a Rota-Baxter algebra and its main characterization by Atkinson's

\footnotetext{
${ }^{1}$ Here the relation is named after the physicists C.-N.Yang and Rodney Baxter.
} 
theorem. Section three contains the main results. We briefly introduce the Hopf algebra of rooted trees and the group of characters and its Lie algebra of infinitesimal characters, with target space of Rota-Baxter type. The Birkhoff decomposition is then derived. We stress that we obtain Bogoliubov's R-bar operation from scratch, as a natural consequence of Atkinson's theorem. We end this work with a summary and an outline describing the case when the Hopf algebra is not cocommutative.

\section{Rota-Baxter algebras and the double con- struction}

Let $\mathbb{K}$ be a field of characteristic 0 . By a $\mathbb{K}$-algebra we mean an associative algebra over $\mathbb{K}$ that is not necessarily unital or commutative unless stated otherwise.

Definition 2.1 Let $\mathcal{A}$ be a $\mathbb{K}$-algebra with a $\mathbb{K}$-linear map $R: \mathcal{A} \rightarrow \mathcal{A}$. We call $\mathcal{A}$ a Rota-Baxter $\mathbb{K}$-algebra and $R$ a Rota-Baxter map (of weight $\theta \in \mathbb{K}$ ) if the operator $R$ holds the following Rota-Baxter relation of weight $\theta \in \mathbb{K}^{2}$ :

$$
R(x) R(y)+\theta R(x y)=R(R(x) y+x R(y)), \forall x, y \in \mathcal{A} .
$$

We note that a Rota-Baxter relation can be defined on $\mathcal{A}$ even when the multiplication on $\mathcal{A}$ is not associative, e.g. when $\mathcal{A}$ is a Lie or pre-Lie algebra. In the case $\theta=0$, the Rota-Baxter operator is somewhat degenerate (see Atkinson's theorem below). When $\theta \neq 0$, a simple transformation $R \rightarrow \theta^{-1} R$ gives the standard form of Eq. (11). For the rest of the paper we will always assume the Rota-Baxter map to be of weight $\theta=1$, i.e. to be in standard form.

Remark 2.2 (1) If $R$ fulfills relation (11) for $\theta=1$ then $\tilde{R}:=i d-R$ fulfills the same Rota-Baxter relation.

(2) The images of $R$ and $i d-R$ give subalgebras in $\mathcal{A}$.

Example 2.3 (1) An important class of Rota-Baxter maps is given by certain projectors. This is the case for the minimal subtraction map $R_{M S}$ in renormalization theory, which is a Rota-Baxter map of weight $\theta=1$ on the algebra of Laurent series $\mathbb{C}\left[\left[\epsilon, \epsilon^{-1}\right][18]\right.$. For $\sum_{k \geq-m}^{\infty} c_{k} \epsilon^{k} \in \mathbb{C}\left[\left[\epsilon, \epsilon^{-1}\right]\right.$ :

$$
R_{m s}\left(\sum_{k \geq-m}^{\infty} c_{k} \epsilon^{k}\right):=\sum_{k \geq-m}^{-1} c_{k} \epsilon^{k}
$$

(2) Another nice example [10] of a Rota-Baxter map of weight $\theta \in \mathbb{K}$ is the operator $\beta: M_{n}^{u p}(\mathbb{K}) \rightarrow M_{n}^{u p}(\mathbb{K})$ defined on the subalgebra of $n \times n$ upper

\footnotetext{
${ }^{2}$ Some authors denote this relation in the form $R(x) R(y)=R(R(x) y+x R(y)+\lambda x y)$. So $\lambda=-\theta$.
} 
triangular matrices $M_{n}^{u p}(\mathbb{K}) \subset M_{n}(\mathbb{K})$, mapping an element $x$ to the diagonal matrices $M_{n}^{u p}(\mathbb{K}) \ni x \mapsto \beta(x) \in M_{n}^{d}(\mathbb{K}) \subset M_{n}^{u p}(\mathbb{K})$ :

$$
(\beta(x))_{i j}:=\delta_{i j} \theta \sum_{k \geq i}^{n} x_{i k} .
$$

(3) The Riemann integral:

$$
R[f](x):=\int_{0}^{x} f(y) d y
$$

provides an example for a Rota-Baxter map of weight zero; Eq. (11) for $\theta=0$ gives the rule for integration by parts.

We now introduce the modified Rota-Baxter relation, its Lie algebraic version can be found in [13, 14].

Definition 2.4 Let $\mathcal{A}$ be a Rota-Baxter algebra, $R$ its Rota-Baxter map. Define the operator $B: \mathcal{A} \rightarrow \mathcal{A}, \quad B:=i d-2 R$ to be the modified RotaBaxter map and call the corresponding relation fulfilled by $B$ :

$$
B(x) B(y)=B(B(x) y+x B(y))-x y, \forall x, y \in \mathcal{A}
$$

the modified Rota-Baxter relation.

Proposition 2.5 In the case of the Rota-Baxter algebra $\mathcal{A}$ to be either an associative or pre-Lie $\mathbb{K}$-algebra, the (modified) Rota-Baxter relation naturally extends to the Lie algebra $\mathcal{L}_{\mathcal{A}}$ with bracket $[x, y]:=x y-y x, \forall x, y \in \mathcal{A}$ :

$$
\begin{aligned}
& {[R(x), R(y)]+R([x, y])=R([R(x), y]+[x, R(y)])} \\
& {[B(x), B(y)]=B([B(x), y]+[x, B(y)])-[x, y] .}
\end{aligned}
$$

The proof of this follows from a simple calculation. The relations (4) and (5) are well-known as the (operator form of the) classical Yang-Baxter and modified Yang-Baxter equation.

The following Proposition 2.6] and Theorem 2.9 characterize Rota-Baxter algebras.

Proposition 2.6 Let $\mathcal{A}$ be a Rota-Baxter algebra with (modified) Rota-Baxter map $R(B=i d-2 R)$. Equipped with the new product:

$$
\begin{aligned}
a *_{R} b & :=R(a) b+a R(b)-a b \\
& =-\frac{1}{2}(B(a) b+a B(b)),
\end{aligned}
$$

$\mathcal{A}$ is again a Rota-Baxter algebra of the same type, denoted by $\mathcal{A}_{R}$.

The proof of this proposition is immediate by the definition of $*_{R}$. We call this new Rota-Baxter algebra $\mathcal{A}_{R}$ the double of $\mathcal{A}$. 
Remark 2.7 (1) It is obvious that Proposition 2.6 implies a whole hierarchy of doubles $\mathcal{A}_{R}^{(i)}\left(\right.$ here, $\left.*_{R}=*_{R}^{(1)}\right)$ :

$$
\begin{gathered}
\mathcal{A}_{R}^{(0)}:=\mathcal{A}, \mathcal{A}_{R}^{(1)}:=\left(\mathcal{A}, *_{R}\right), \cdots, \mathcal{A}_{R}^{(i)}:=\left(\mathcal{A}, *_{R}^{(i)}\right), \cdots \\
a *_{R}^{(i)} b:=\frac{d^{i}}{d t^{i}}{ }_{t=0} e^{-\frac{1}{2} t B}(a) e^{-\frac{1}{2} t B}(b), a, b \in \mathcal{A} .
\end{gathered}
$$

Let us call $\mathcal{A}_{R}^{(i)}$ the i-th double of $\mathcal{A}$ and the double of $\mathcal{A}_{R}^{(i-1)}$.

(2) The Rota-Baxter map becomes an algebra homomorphism between $\mathcal{A}_{R}^{(i)}$ and $\mathcal{A}_{R}^{(i-1)}, i \in \mathbb{N}$ :

$$
R\left(a *_{R}^{(i)} b\right)=R(a) *_{R}^{(i-1)} R(b) .
$$

(3) For the Rota-Baxter map $\tilde{R}:=i d-R$, we have

$$
\tilde{R}\left(a *{ }_{R}^{(i)} b\right)=-\tilde{R}(a) *_{R}^{(i-1)} \tilde{R}(b) .
$$

The last equation can be written as $\hat{R}:=-\tilde{R}, \hat{R}\left(a *_{R}^{(i)} b\right)=\hat{R}(a) *_{R}^{(i-1)} \hat{R}(b)$. As a side remark we should mention that the notion of the double of $\mathcal{A}$ for associative and non-associative algebras may be found in 14 .

A relation closely related to the Rota-Baxter relation (11) is:

$$
N(x) N(y)+N^{2}(x y)=N(N(x) y+x N(y)), \quad x, y \in \mathcal{A} .
$$

The map $N$ might be called an associative Niejnhuis operator or just Nijenhuis map for short [28]. In this setting "associative" refers to the relation (86) to distinguish it clearly form its Lie algebraic version [29, 30]:

$$
[N(x), N(y)]+N^{2}([x, y])=N([N(x), y]+[x, N(y)]) .
$$

As in the case of the Rota-Baxter relation, a Niejnhuis map on a $\mathbb{K}$-algebra $\mathcal{A}$ also gives a Niejnhuis map for the associated Lie algebra $\mathcal{L}_{\mathcal{A}}:=(\mathcal{A},[-,-])$, $[-,-]$ being the commutator. Also, similar to the case of the Rota-Baxter relation the associative Niejnhuis identity implies a hierarchy of algebra products. We will not go further into details with respect to this relation.

Proposition 2.8 Let $\mathcal{A}$ be a commutative, associative Rota-Baxter algebra. For $n \in \mathbb{N}, x \in \mathcal{A}$ we have

$$
\begin{aligned}
(-R(x))^{n} & =-R\left(x^{n}+\sum_{k=1}^{n-1}\left(\begin{array}{l}
n \\
k
\end{array}\right)(-R(x))^{(n-k)} x^{k}\right), \\
\tilde{R}(x)^{n} & =\tilde{R}\left(x^{n}+\sum_{k=1}^{n-1}\left(\begin{array}{l}
n \\
k
\end{array}\right)(-R(x))^{(n-k)} x^{k}\right) .
\end{aligned}
$$

The proof works inductively. Proposition [2.8, and equations (10, 11) will lead us to the twisted antipode formula [17, 18, 19.

We come now to the important result of Atkinson, characterizing RotaBaxter algebras. 
Theorem 2.9 (Atkinson [2]): For a $\mathbb{K}$-algebra $\mathcal{A}$ with a linear map $R: \mathcal{A} \rightarrow$ $\mathcal{A}$ to be a Rota-Baxter $\mathbb{K}$-algebra, it is necessary and sufficient that $\mathcal{A}$ has a subdirect Birkhoff decomposition.

It should be underlined here that this theorem is true quite generally, in the sense that the algebra needs not to be associative, nor commutative.

Essentially, the subdirect Birkhoff decomposition in this case means that the cartesian product $\mathcal{D}:=(R(\mathcal{A}),-\tilde{R}(\mathcal{A})) \subset \mathcal{A} \times \mathcal{A}$ is a subalgebra in $\mathcal{A} \times \mathcal{A}$ and that every element $x \in \mathcal{A}$ has a unique decomposition $x=R(x)+\tilde{R}(x)$. The double construction introduced here and Atkinson's theorem should be compared with the results in [13, 14, 15, 16].

\section{R-matrix approach to renormalization: the rooted ladder tree case}

We will now briefly introduce the Connes-Kreimer Hopf algebra of rooted trees [22, 23, 24].

Definition 3.1 A rooted tree is a finite, connected oriented graph without loops in which every vertex has exactly one incoming edge, except one (the root) which has no incoming but only outgoing edges. We denote the set of edges and vertices of a rooted tree $T$ by $E(T), V(T)$ respectively.

Let us denote the set of (isomorphism classes of) rooted trees by $\mathcal{T}_{r t}$.

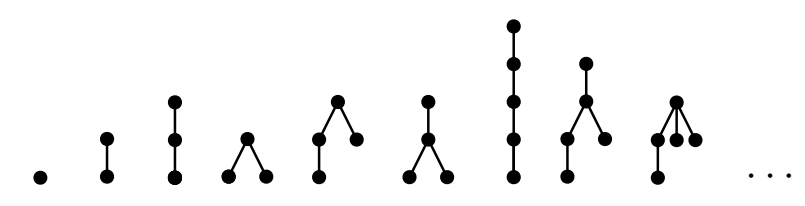

Let $\mathcal{H}_{r t}$ be the commutative algebra generated by these symbols $T \in \mathcal{T}_{r t}$ (one for each isomorphism class). The commutative product $m_{\mathcal{H}_{r t}}: \mathcal{H}_{r t} \otimes$ $\mathcal{H}_{r t} \rightarrow \mathcal{H}_{r t}$ is written as concatenation $m_{\mathcal{H}_{r t}}\left(T^{\prime}, T^{\prime \prime}\right)=: T^{\prime} T^{\prime \prime}$ and the empty tree is denoted by 1 giving the unit. The algebra may be graded by the number of vertices $\#(T)=|V(T)|$ of the rooted tree $T$. We equip this algebra with a counit map $\epsilon: \mathcal{H}_{r t} \rightarrow \mathbb{K}, \epsilon(1):=1$ and $\epsilon\left(T_{1} \cdots T_{n}\right)=0$ for $T_{1} \cdots T_{n} \neq 1$

We now define the coproduct $\Delta: \mathcal{H}_{r t} \rightarrow \mathcal{H}_{r t} \otimes \mathcal{H}_{r t}$. For this we first introduce the notion of simple cuts on rooted trees. A simple or admissible cut $c_{T}$ of a tree $T$ is a subset of its edges such that along any path from its root to one of its leaves one meets at most one element of $c_{T}$. Deleting the set $c_{T} \subset E(T)$ of edges in $T$ produces one tree $R_{c_{T}}$ still containing the original root and a set of pruned rooted trees $P_{c_{T}}$, the roots of which are identified with the vertex which had the cut edge in $c_{T}$ as incoming edge. The following examples may be helpful in understanding the concept of simple cuts, $R_{c_{T}}$ and $P_{c_{T}}$ : 


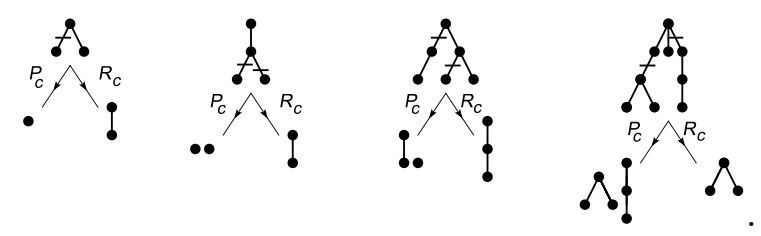

The coproduct may then be defined as follows. Let $C_{T}$ be the set of all admissible cuts of the rooted tree $T$. We exclude the empty cut $c_{T}^{(0)}, P_{c_{T}^{(0)}}=$ $\emptyset, R_{c_{T}^{(0)}}=T$ and the full cut $c_{T}^{(1)}, P_{c_{T}^{(1)}}=T, R_{c_{T}^{(1)}}=\emptyset$ :

$$
\Delta(T)=T \otimes 1+1 \otimes T+\sum_{c_{T} \in C_{T}} P_{c_{T}}\left(T^{\prime}\right) \otimes R_{c_{T}}\left(T^{\prime \prime}\right) .
$$

Here $T^{\prime}$, respectively $T^{\prime \prime}$ stand for the rooted trees produced when applying $c_{T}$. As an example we calculate the coproduct of the rooted tree $\dot{\lambda}$ of weight $\#(\AA)=4$ :

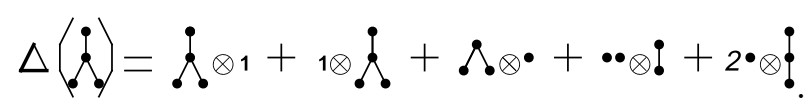

The coproduct (12) is extended by linearity, and we define it to be an algebra homomorphism $\Delta\left(T_{1} \cdots T_{n}+T_{n+1} \cdots T_{m}\right)=\prod_{i=1}^{n} \Delta\left(T_{i}\right)+\prod_{i=n+1}^{m} \Delta\left(T_{i}\right)$, $\Delta(1):=1 \otimes 1$. Obviously, (12) is not cocommutative. So far we have a connected graded bialgebra, hence by general arguments a rooted tree Hopf algebra with antipode $S: \mathcal{H}_{r t} \rightarrow \mathcal{H}_{r t}$ :

$$
S(T):=-T-\sum_{c_{T} \in C_{T}} S\left(P_{c_{T}}\left(T^{\prime}\right)\right) R_{c_{T}}\left(T^{\prime \prime}\right) .
$$

Again we exclude the empty and full cut, i.e. $c_{T}^{(0)}, c_{T}^{(1)}$ respectively, in the above sum.

The Hopf algebra $\mathcal{H}_{r t}$ contains a cocommutative Hopf subalgebra denoted by $\mathcal{H}_{l r t}$, generated by rooted ladder graphs:

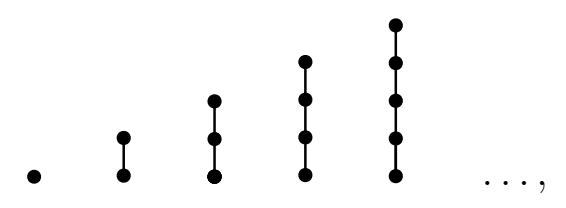

which we will denote in general by $t_{n} \in \mathcal{H}_{l r t} \subset \mathcal{H}_{r t}$, where $n \in \mathbb{N}$ is the number of vertices, having at most one incoming and also at most one outgoing edge. By $t_{0}$ we denote the unit, i.e. the empty tree 1 . The coproduct then becomes:

$$
\Delta\left(t_{n}\right)=t_{n} \otimes 1+1 \otimes t_{n}+\sum_{i=1}^{n-1} t_{i} \otimes t_{n-i} .
$$

The graded dual $\mathcal{H}_{r t}^{*}$ equipped with the convolution product $f \star g:=m_{\mathbb{K}} \circ f \otimes$ $g \circ \Delta, f, g \in \mathcal{H}_{r t}^{*}$ becomes an associative $\mathbb{K}$-algebra. We denote the pairing by brackets, $\langle f, T\rangle:=f(T) \in \mathbb{K}$. 
Let $\operatorname{char}_{\mathbb{K}} \mathcal{H}_{r t} \subset \mathcal{H}_{r t}^{*}$ be the group of characters, i.e. algebra morphisms into $\mathbb{K}$, with inverse $\phi^{-1}:=\phi \circ S, \phi \in \operatorname{char}_{\mathbb{K}} \mathcal{H}_{r t}$. Let $\partial \operatorname{char}_{\mathbb{K}} \mathcal{H}_{r t} \subset \mathcal{H}_{r t}^{*}$ be the Lie algebra of infinitesimal characters, i.e. derivations into $\mathbb{K}$ :

$$
Z\left(T^{\prime} T^{\prime \prime}\right)=Z\left(T^{\prime}\right) \epsilon\left(T^{\prime \prime}\right)+\epsilon\left(T^{\prime}\right) Z\left(T^{\prime \prime}\right), Z \in \partial \operatorname{char}_{\mathbb{K}} \mathcal{H}_{r t} .
$$

with Lie bracket:

$$
\left[Z^{\prime}, Z^{\prime \prime}\right]:=Z^{\prime} \star Z^{\prime \prime}-Z^{\prime \prime} \star Z^{\prime} .
$$

$\partial c h a r_{\mathbb{K}} \mathcal{H}_{r t}$ is generated by the infinitesimal characters $Z_{T}$ indexed by rooted trees $T \in \mathcal{T}_{r t}$, and defined by:

$$
\left\langle Z_{T}, T^{\prime}\right\rangle:=\delta_{T, T^{\prime}}
$$

Tree monomials are excluded from the index set due to the Leibniz rule (15). The Lie bracket for these generators is given by [22]:

$$
\left[Z_{T^{\prime}}, Z_{T^{\prime \prime}}\right]=\sum_{T \in \mathcal{T}_{r t}}\left(n\left(T^{\prime}, T^{\prime \prime} ; T\right)-n\left(T^{\prime \prime}, T^{\prime} ; T\right)\right) Z_{T},
$$

where the $n\left(T^{\prime}, T^{\prime \prime} ; T\right) \in \mathbb{N}$ denote so-called section coefficients which count the number of single simple cuts, $\left|c_{T}\right|=1$, such that $P_{c_{T}}=T^{\prime}$ and $R_{c_{T}}=T^{\prime \prime}$ :

$$
\begin{aligned}
{\left[Z_{\bullet}, Z_{\mathfrak{\bullet}}\right]=} & Z_{\mathfrak{!}}+2 Z_{\boldsymbol{\Lambda}}-Z_{\mathfrak{!}}=2 Z_{\boldsymbol{\Lambda}} \\
{\left[Z_{\boldsymbol{\Lambda}}, Z_{\bullet}\right]=} & \frac{1}{2}\left[\left[Z_{\bullet}, Z_{\mathfrak{!}}\right], Z_{\bullet}\right] \\
= & Z_{\mathfrak{\iota}}-3 Z_{\boldsymbol{\Lambda}}-Z_{\boldsymbol{\Lambda}}
\end{aligned}
$$

Remark 3.2 (1) The composition $Z_{T^{\prime}} \star Z_{T^{\prime \prime}}=\sum_{T \in \mathcal{T}_{r t}} n\left(T^{\prime}, T^{\prime \prime} ; T\right) Z_{T}$ defines a left pre-Lie algebra structure on $\partial$ char $_{\mathbb{K}} \mathcal{H}_{r t}$.

(2) Antisymmetrizing this pre-Lie product gives the above Lie algebra, which lies at the heart of the combinatorics of renormalization theory in pQFT [25, 26].

The exponential map gives the bijection from $\operatorname{cchar}_{\mathbb{K}} \mathcal{H}_{r t} \rightarrow \operatorname{char}_{\mathbb{K}} \mathcal{H}_{r t}$ :

$$
\exp ^{\star}(Z):=\sum_{n=0}^{\infty} \frac{Z^{\star n}}{n !} \in \operatorname{char}_{\mathbb{K}} \mathcal{H}_{r t} .
$$

Where $Z$ is given as a series

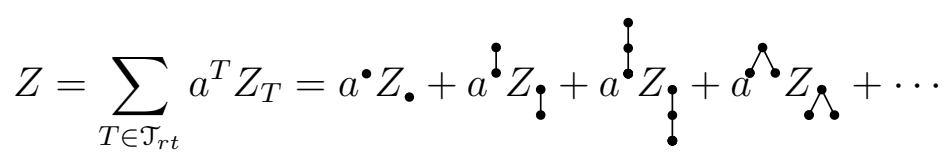

The exponential map (21) is well defined since due to the Leibniz rule (15) we have $Z^{\star n}(T)=0$ for $n>\#(T)$. 
Following the Hopf algebraic approach to renormalization in perturbative quantum field theory (pQFT), we introduce the notion of regularized (infinitesimal) characters, mapping $\mathcal{H}_{r t}$ into a commutative, associative, unital Rota-Baxter algebra $\mathcal{A}^{3}$. We therefore extend $\mathcal{H}_{r t}^{*}$ to $\mathcal{H}_{r t}^{*} \otimes \mathcal{A}=L\left(\mathcal{H}_{r t}, \mathcal{A}\right)$, consisting of $\mathbb{K}$-linear maps from $\mathcal{H}_{r t}$ into the Rota-Baxter algebra $\mathcal{A}$, i.e. $\langle\phi, T\rangle \in \mathcal{A}, \phi \in L\left(\mathcal{H}_{r t}, \mathcal{A}\right), T \in \mathcal{H}_{r t}$.

We then lift the Rota-Baxter map $R: \mathcal{A} \rightarrow \mathcal{A}$ to $L\left(\mathcal{H}_{r t}, \mathcal{A}\right)$.

Proposition 3.3 Define the linear map $\mathcal{R}: L\left(\mathcal{H}_{r t}, \mathcal{A}\right) \rightarrow L\left(\mathcal{H}_{r t}, \mathcal{A}\right)$ by $f \mapsto \mathcal{R}(f):=R \circ f: \mathcal{H}_{r t} \rightarrow R(\mathcal{A})$. Then $L\left(\mathcal{H}_{r t}, \mathcal{A}\right)$ becomes an associative, unital Rota-Baxter algebra. The Lie algebra of infinitesimal characters $\mathcal{L}_{\mathcal{H}_{r t}^{*}} \subset L\left(\mathcal{H}_{r t}, \mathcal{A}\right)$ with bracket (16) becomes a Lie Rota-Baxter algebra, i.e. for $Z^{\prime}, Z^{\prime \prime} \in \partial \operatorname{char}_{\mathcal{A}} \mathcal{H}_{r t}$,

$$
\left[\mathcal{R}\left(Z^{\prime}\right), \mathcal{R}\left(Z^{\prime \prime}\right)\right]=\mathcal{R}\left(\left[Z^{\prime}, \mathcal{R}\left(Z^{\prime \prime}\right)\right]\right)+\mathcal{R}\left(\left[\mathcal{R}\left(Z^{\prime}\right), Z^{\prime \prime}\right]\right)-\mathcal{R}\left(\left[Z^{\prime}, Z^{\prime \prime}\right]\right) .
$$

Notice that we replaced $\mathbb{K}$ by $\mathcal{A}$ for the target space of the regularized infinitesimal characters. The proof follows from the fact that $R$ is $\mathbb{K}$-linear and:

$$
\begin{aligned}
\mathcal{R}(f) \star \mathcal{R}(g)(T)= & m_{\mathcal{A}}(\mathcal{R}(f) \otimes \mathcal{R}(g)) \circ \Delta(T) \\
= & m_{\mathcal{A}}\left(R\left(f\left(T_{(1)}\right)\right) \otimes R\left(g\left(T_{(2)}\right)\right)\right) \\
\equiv & -R \circ m_{\mathcal{A}}\left(f\left(T_{(1)}\right) \otimes g\left(T_{(2)}\right)\right)+ \\
& R \circ m_{\mathcal{A}}\left(f\left(T_{(1)}\right) \otimes R\left(g\left(T_{(2)}\right)\right)\right)+ \\
& R \circ m_{\mathcal{A}}\left(R\left(f\left(T_{(1)}\right)\right) \otimes g\left(T_{(2)}\right)\right) \\
= & -R \circ m_{\mathcal{A}}(f \otimes g) \circ \Delta(T)+\quad \\
R \circ m_{\mathcal{A}}(f \otimes \mathcal{R}(g)) \circ \Delta(T)+ & R \circ m_{\mathcal{A}}(\mathcal{R}(f) \otimes g) \circ \Delta(T) \\
= & \mathcal{R}(f \star \mathcal{R}(g))(T)+\mathcal{R}(\mathcal{R}(f) \star g)(T)-\mathcal{R}(f \star g)(T),(23)
\end{aligned}
$$

where we used Sweedler's notation $\Delta(T)=\sum T_{(1)} \otimes T_{(2)}$ for the coproduct (12), omiting the summation sign above. For the second assertion, we only have to show that $\mathcal{R}: \partial \operatorname{char}_{\mathcal{A}} \mathcal{H}_{r t} \rightarrow \partial \operatorname{char}_{\mathcal{A}} \mathcal{H}_{r t}$, but this again follows from the $\mathbb{K}$-linearity of $R$ and $\epsilon(T) \in \mathbb{K}$.

Using the double construction and Atkinson's theorem of section 2 we have the following

Lemma 3.4 The Rota-Baxter algebra $L\left(\mathcal{H}_{r t}, \mathcal{A}\right)$ equipped with the convolution product:

$$
f \star_{\mathcal{R}} g=f \star \mathcal{R}(g)+\mathcal{R}(f) \star g-f \star g
$$

gives a Rota-Baxter algebra structure on the set of linear functionals into the double $\mathcal{A}_{R}$ of $\mathcal{A}$, denoted by $L\left(\mathcal{H}_{r t}, \mathcal{A}_{R}\right)$. An analog for $\mathcal{L}_{\mathcal{H}_{r t}^{*}}$ exists and is

\footnotetext{
${ }^{3} \mathcal{A}=\mathbb{C}\left[\left[\epsilon, \epsilon^{-1}\right]\right.$ in dimensional regularization together with minimal subtraction in pQFT, where the Rota-Baxter $R$ map is then given by equation (2)
} 
denoted by $\mathcal{L}_{\mathcal{H}_{r t}^{*} \mathcal{R}} \cdot \mathcal{R}$ becomes a (Lie) algebra morphism $\left(\mathcal{L}_{\mathcal{H}_{r t}^{*} \mathcal{R}} \rightarrow \mathcal{L}_{\mathcal{H}_{r t}^{*}}\right)$ $L\left(\mathcal{H}_{r t}, \mathcal{A}_{R}\right) \rightarrow L\left(\mathcal{H}_{r t}, \mathcal{A}\right)$.

Remark 3.5 The above is also true for $\tilde{R}:=i d-R$, respectively $\tilde{\mathcal{R}}$ (see remark 2.7). We will denote $\mathcal{R}\left(\mathcal{L}_{\mathcal{H}_{r t}^{*}}\right)$ by $\mathcal{L}_{\mathcal{F}_{r t}^{*}}^{-}$and $\tilde{\mathcal{R}}\left(\mathcal{L}_{\mathcal{H}_{r t}^{*}}\right)$ by $\mathcal{L}_{\mathcal{H}_{r t}^{*}}^{+}$.

We now apply Atkinson's theorem to the Lie algebra $\mathcal{L}_{\mathcal{H}_{r t}^{*}}$ of infinitesimal characters, the generators of the group of Hopf algebra characters char ${ }_{\mathcal{A}} \mathcal{H}_{r t}$.

Lemma 3.6 Every infinitesimal character $Z \in \mathcal{L}_{\mathcal{H}_{r t}^{*}}$ has a unique subdirect Birkhoff decomposition $Z=\mathcal{R}(Z)+\tilde{\mathcal{R}}(Z)$.

Remark 3.7 (1) In the case of an idempotent Rota-Baxter map $R$ we have a direct decomposition $\mathcal{A}=\mathcal{A}_{-}+\mathcal{A}_{+}$respectively $\mathcal{L}_{\mathcal{H}_{r t}^{*}}=\mathcal{L}_{\mathcal{H}_{r t}^{*}}^{-}+\mathcal{L}_{\mathcal{F}_{r t}^{*}}^{+}$.

(2) Let $Z \in \mathcal{L}_{\mathcal{H}_{r t}^{*}}$ be the infinitesimal character generating the character $\phi=$ $\exp ^{\star}(Z) \in \operatorname{char}_{\mathcal{A}} \mathcal{H}_{r t}$. Using the result in proposition 2.8, easily generalized to the non-commutative case, we then see that $\exp ^{\star}(\mathcal{R}(Z))=\mathcal{R}\left(\exp ^{\star \mathcal{R}}(Z)\right)$.

Let us define

$$
b[\phi]:=\exp ^{\star \mathcal{R}}(Z)
$$

which is a character of $\mathcal{H}_{r t} \rightarrow \mathcal{A}_{R}$, i.e. $b[\phi] \in \operatorname{char}_{\mathcal{A}_{R}} \mathcal{H}_{r t}$ and which we will call Bogoliubov's recursion $\bar{R}$-map for reasons which will become clear soon. Therefore

$$
\begin{aligned}
\exp ^{\star}(\mathcal{R}(Z))\left(T^{\prime} T^{\prime \prime}\right) & =\mathcal{R}\left(\exp ^{\star \mathcal{R}}(Z)\right)\left(T^{\prime} T^{\prime \prime}\right) \\
& =R\left(b[\phi]\left(T^{\prime}\right) *_{R} b[\phi]\left(T^{\prime \prime}\right)\right) \\
& =\exp ^{\star}(\mathcal{R}(Z))\left(T^{\prime}\right) \exp ^{\star}(\mathcal{R}(Z))\left(T^{\prime \prime}\right) .
\end{aligned}
$$

We then have

Lemma 3.8 The Lie Rota-Baxter map $\mathcal{R}(\tilde{\mathcal{R}})$ becomes a Lie group (anti-) homomorphism from char ${ }_{\mathcal{A}_{R}} \mathcal{H}_{r t}$ to char ${ }_{\mathcal{A}}^{-(+)} \mathcal{H}_{r t}$, where char $_{\mathcal{A}}^{-(+)} \mathcal{H}_{r t}$ are the Lie subgroups generated by the Lie subalgebras $\mathcal{L}_{\mathcal{H}_{r t}^{*}}^{-(+)}$.

As we already mentioned in the introduction, here we will only consider in detail the simple case of the cocommutative Hopf subalgebra $\mathcal{H}_{l r t}$, respectively $L\left(\mathcal{H}_{l r t}, \mathcal{A}\right)$. The latter is generated by the $Z_{t_{n}} \in \partial \operatorname{char}_{\mathcal{A}} \mathcal{H}_{r t}, n \in \mathbb{N}$ and necessarily is an abelian Lie algebra $\mathcal{L}_{\mathcal{H}_{l r t}^{*}},\left[Z_{t_{n}}, Z_{t_{m}}\right]=0, n, m \in \mathbb{N}$.

The abelianess of $\mathcal{L}_{\mathcal{H}_{l r t}^{*}}$ and Atkinson's result imply the following theorem (which extends to the non-abelian case using the appropriate $\mathrm{BCH}$ formulas for the (multi-)commutator of $\mathcal{R}(Z)$ with $\tilde{\mathcal{R}}(Z)$ ).

Theorem 3.9 (Ladder case of integral renormalization): Let $\phi \in \operatorname{char}_{\mathcal{A}} \mathcal{H}_{l r t}$ be generated by $Z \in \mathcal{L}_{\mathcal{H}_{l t}^{*}}$, i.e. $\exp ^{\star}(Z)=\phi$. We have the following factorization:

$$
\begin{aligned}
\exp ^{\star}(Z)=\phi & =\exp ^{\star}(\mathcal{R}(Z)+\tilde{\mathcal{R}}(Z)) \\
& =\exp ^{\star}(\mathcal{R}(Z)) \star \exp ^{\star}(\tilde{\mathcal{R}}(Z)) .
\end{aligned}
$$


Proposition 3.10 With the same assumption as in theorem 3.9 and the definitions $\phi_{-}^{-1}:=\exp ^{\star}(\mathcal{R}(Z))$ and $\phi_{+}:=\exp ^{\star}(\tilde{\mathcal{R}}(Z))$, we have

$$
\begin{aligned}
\phi_{-}\left(t_{n}\right) & =\exp ^{\star}(-\mathcal{R}(Z))\left(t_{n}\right) \\
& =\mathcal{R}\left(\exp ^{\star \mathcal{R}}(-Z)\right)\left(t_{n}\right) \\
& =-\mathcal{R}\left\{\phi\left(t_{n}\right)+\sum_{k=1}^{n-1} \phi_{-}\left(t_{k}\right) \phi\left(t_{n-k}\right)\right\} \\
\phi_{+}\left(t_{n}\right) & =\exp ^{\star}(\tilde{\mathcal{R}}(Z))\left(t_{n}\right) \\
& =-\tilde{\mathcal{R}}\left(\exp ^{\star \mathcal{R}}(-Z)\right)\left(t_{n}\right) \\
& =\tilde{\mathcal{R}}\left\{\phi\left(t_{n}\right)+\sum_{k=1}^{n-1} \phi_{-}\left(t_{k}\right) \phi\left(t_{n-k}\right)\right\} .
\end{aligned}
$$

The proof of this proposition follows immediately by proposition 2.8 and equations (10, 11). It can be generalized to the non-abelian case using the Hochschild cohomology of the Hopf algebra as in [27] and the resolution of the non-abelian Lie algebra in terms of its lower central series.

Remark 3.11 (1) From expressions (25, 27, 29) it is evident that $\phi_{ \pm}$are characters. We will see the same for the general case.

(2) For the ladder case we therefore arrive at the following result. Since $\exp ^{\star}(-\mathcal{R}(Z))=-\mathcal{R}(b[\phi])$ (remark 3.7) we have

$$
b[\phi]\left(t_{n}\right)=\exp ^{\star \mathcal{R}}(Z)\left(t_{n}\right)=\phi\left(t_{n}\right)+\sum_{k=1}^{n-1} \phi_{-}\left(t_{k}\right) \phi\left(t_{n-k}\right) .
$$

This justifies the name Bogoliubov's $\bar{R}$-map [19] for $\exp ^{\star_{\mathcal{R}}}(Z)$, which finds its natural algebraic formulation as a character $\exp ^{\star \mathcal{R}}(Z) \in \operatorname{char}_{\mathcal{A}_{R}} \mathcal{H}_{l r t}$ and which is mapped by the Rota-Baxter operator $\mathcal{R}$ into $\operatorname{char}_{\mathcal{A}} \mathcal{H}_{l r t}$. As mentioned before this result carries over to the general case, i.e. to the non-cocommutative Hopf algebras of Feynman graphs or arbitrary decorated rooted trees. Note that formulas (28, 30) have been established already in [17, while to express (27, 29) in a convenient way using the necessary $\mathrm{BCH}$ corrections will be reserved to future work.

Also, we would like to underline the similarity with the factorization theorems in 14, 15, 16. We will dwell on this connection in greater depth in the future.

Let us summarize the result in proposition 3.10. The (abelian) Lie algebra $\mathcal{L}_{\mathcal{H}_{l t}^{*}}$ naturally extends to a Lie Rota-Baxter algebra due to the Rota-Baxter structure on its target space. Therefore it contains two Lie algebra structures with respect to the original Lie bracket and the double coming from the Rota-Baxter map R. Due to Atkinson's theorem it decomposes into two Lie subalgebras $\mathcal{L}_{\mathcal{H}_{l r t}^{*}}^{-(+)}$which generate the Lie subgroups $\operatorname{char}_{\mathcal{A}}^{-(+)} \mathcal{H}_{l r t}$. The infinitesimal decomposition on $\mathcal{L}_{\mathcal{H}_{l r t}^{*}}$ extends in the ladder case to the global 
factorization on the Lie group $\operatorname{char}_{\mathcal{A}} \mathcal{H}_{l r t}$. We have the following diagrams on the Lie algebra level, respectively Lie group level.

Let $b$ denote Bogoliubov's recursion formula, which is defined in terms of the exponential with respect to the double product $\star_{\mathcal{R}}$. We define $\mathcal{G}^{l}:=$ $\operatorname{char}_{\mathcal{A}} \mathcal{H}_{l r t}, \mathcal{G}_{\mathcal{R}}^{l}:=\operatorname{char}_{\mathcal{A}_{R}} \mathcal{H}_{l r t}$ and $\mathcal{G}^{l \pm}:=\operatorname{char}_{\mathcal{A}}^{ \pm} \mathcal{H}_{l r t}:$

$$
\mathcal{L}_{\mathcal{H}_{l r t}^{*} \mathcal{R}} \stackrel{(\mathcal{R}, \mathcal{R}-i d)}{\longrightarrow}\left(\mathcal{L}_{\mathcal{H}_{l r t}^{*}}^{-}, \mathcal{L}_{\mathcal{H}_{l r t}^{*}}^{+}\right) \stackrel{(i d,-i d)}{\longrightarrow} \mathcal{L}_{\mathcal{H}_{r t}^{*}}
$$

Here, $Z=\mathcal{R}(Z)-(\mathcal{R}-i d)(Z)=(i d,-i d) \circ(\mathcal{R}, \mathcal{R}-i d)(Z)$ gives the infinitesimal factorization. i.e. Atkinson's theorem.

$$
\mathcal{G}^{l} \stackrel{b}{\rightarrow} \mathcal{G}_{\mathcal{R}}^{l} \stackrel{(-\mathcal{R} \otimes \tilde{\mathcal{R}})}{\longrightarrow}\left(\mathcal{G}^{l-}, \mathcal{G}^{l+}\right) \stackrel{m_{\mathcal{G}^{l}}}{\longrightarrow} \mathcal{G}^{l}
$$

The last diagram contains the global factorization, i.e. on the level of the Lie group $\mathcal{G}^{l}$ coming form the Lie algebra $\mathcal{L}_{\mathcal{H}_{l r t}^{*}}$.

Remark 3.12 (1) Using the idea of normal coordinates in 31 we may relate the simple rooted ladder graphs, given by Schur polynomials $P^{(n)}\left(t_{1}, \ldots, t_{n}\right)^{4}$ of order $n$ for each rooted ladder tree $t_{n}$ and the $\phi_{ \pm}$character in the following way. Starting with the regularized character $\phi: \mathcal{H}_{r t} \rightarrow \mathcal{A}$, we define the series (we omit tensor product signs)

$$
Z_{\phi}:=\sum_{n>0} Z_{t_{n}} \phi\left(P^{(n)}\left(t_{1}, \ldots, t_{n}\right)\right) \in \operatorname{char}_{\mathbb{K}} \mathcal{H}_{r t} \otimes \mathcal{A}
$$

It then follows from 31] that $\exp ^{\star}\left(Z_{\phi}\right)\left(t_{n}\right)=\phi\left(t_{n}\right)$, i.e. $Z_{\phi}$ is the infinitesimal character generating the Lie group element $\phi$. As an example we calculate $\phi_{-}(\vdots)$ :

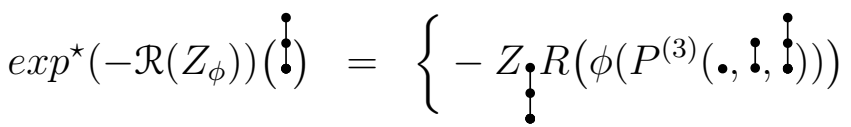

$$
\begin{aligned}
& +\frac{1}{2}\left(Z_{\bullet} \star Z_{\mathfrak{\bullet}}+Z_{\bullet} \star Z_{\bullet}\right) R(\phi(\bullet)) R\left(\phi\left(P^{(2)}(\bullet, \mathfrak{\bullet})\right)\right) \\
& \left.+\frac{-1}{3 !} Z \bullet \star Z \bullet \star Z \cdot R(\phi(\bullet))^{3}\right\}(!) \\
& =-R\left(\phi\left(P^{(3)}(\bullet, \mathfrak{\bullet}, \mathfrak{\bullet})\right)-R(\phi(\bullet)) R\left(\phi\left(P^{(2)}(\bullet, \mathfrak{\bullet})\right)\right)\right. \\
& \left.+\frac{1}{3 !} R(\phi(\bullet))^{3}\right) \text {. }
\end{aligned}
$$

(2) Let us briefly outline the approach to the general case. The full Lie algebra of infinitesimal characters $\mathcal{L}_{\mathcal{H}_{r t}^{*}}$ is of course non-abelian and therefore

\footnotetext{
${ }^{4}$ Schur polynomial of order $n, P^{(n)}$ : Taylor expansion of $\log \left(\sum_{n \geq 0} t_{n} x^{n}\right)=$ $\sum_{n \geq 0} P^{(n)}\left(t_{1}, \ldots, t_{n}\right) x^{n} 31$
} 
the factorization has to include contributions in a subtractive manner to eliminate $\mathrm{BCH}$ terms. This may be achieved in a systematic way using the Baker-Campbell-Hausdorff (BCH) functional

$$
B C H(A, B):=\frac{1}{2}[A, B]+\frac{1}{12}([A,[A, B]]-[B,[A, B]])+\cdots
$$

which starts with a commutator, i.e. is of order $>1$ in the number of infinitesimal characters. Recursively, order by order in the grading on $\mathcal{H}_{r t}$, the correct contributions may be calculated. The first term of second order essentially takes care of the cherry tree $\triangle$ renormalization:

$$
\chi^{(2)}=-\frac{1}{2}[\mathcal{R}(Z), Z]
$$

for $\phi=\exp ^{\star}(Z) \in \operatorname{char}_{\mathcal{A}} \mathcal{H}_{r t}$ and which should be compared to (19). Again, the normal coordinates theorem in 31] provides a convenient way to identify terms from the $\mathrm{BCH}$ formula. This way we derive the implicitly given formulae for $\phi_{ \pm}$in [19], respectively give an explicit formula for them in terms of the exponential map and an element in the image of the Rota-Baxter maps $\mathcal{R}$, respectively $\tilde{\mathcal{R}}$.

(3) The presented setting resembles the loop algebra-group case of integrable systems theory. The generalization takes place by using a general RotaBaxter algebra as the target space. In a later publication we will dwell more carefully on this point.

(4) Following the recent work by Sakakibara [32] we derive the scattering type formula for $\phi_{ \pm}$. We first extend the Lie algebra $\partial c h a r_{\mathcal{A}} \mathcal{H}_{r t}$ by an element $Z_{0}$ such that $\left[Z_{0}, Z_{T}\right]=Y\left(Z_{T}\right):=\#(T) Z_{T}$ where $Y$ is the grading operator $Y(T):=\#(T)$, i.e. a derivation on $\mathcal{H}_{r t}$ (see [20] for details). This implies an one parameter group $\theta_{t} \in \operatorname{Aut}\left(\mathcal{H}_{r t}\right)$ acting on $\operatorname{char}_{\mathcal{A}} \mathcal{H}_{r t}$ by

$$
\left\langle\theta_{t}(\phi), T\right\rangle:=\left\langle\phi, \theta_{t}(T)\right\rangle,
$$

i.e. $\theta_{t}=A d_{\exp ^{\star}\left(t Z_{0}\right)}$, and such that $\left.\frac{d \theta_{t}}{d t}\right|_{t=0}=Y$. We then define a so-called $\beta$-function:

$$
\begin{aligned}
\beta(\phi) & :=\phi_{ \pm} \star Y\left(\phi_{ \pm}^{-1}\right) \\
& =\phi_{ \pm} \star\left[Z_{0}, \phi_{ \pm}^{-1}\right]=\phi_{ \pm} \star Z_{0} \star \phi_{ \pm}^{-1}-Z_{0},
\end{aligned}
$$

such that 32

$$
\begin{array}{ccc}
\exp ^{\star}\left(t\left(\beta(\phi)+Z_{0}\right)\right) \star \exp ^{\star}\left(-t Z_{0}\right) & =\phi_{ \pm} \star \theta_{t}\left(\phi_{ \pm}^{-1}\right) \\
\stackrel{t \rightarrow \infty}{\longrightarrow} & \phi_{ \pm} .
\end{array}
$$

This should be compared to the expression we found for $\phi_{ \pm}$in terms of the Rota-Baxter map $\mathcal{R}$. 


\section{Summary, Conclusion and Outlook}

In earlier work [19] the combinatorics of renormalization in pQFT was described in terms of a Birkhoff factorization of the regularized Hopf algebra characters. The identification of the singular part $\phi_{-}$as a character relies on the Rota-Baxter structure on the target space of the characters. Emphasizing the latter point and restricting for pedagogical reasons to the simple case of rooted ladder trees we were able to derive the twisted antipode formula for the singular part of the Birkhoff decomposition, solely using the properties of the Rota-Baxter map lifted to the Lie algebra of infinitesimal characters.

Extending this simple exercise to the general case of a non-cocommutative Hopf algebra poses no conceptual challenge but becomes technically more demanding and will be presented elsewhere.

In our view it is important to underline that it is the Lie algebra of rooted trees, or more generally the insertion-elimination Feynman Lie algebra, which completely describes the process of renormalization in pQFT. This will become even more apparent when we treat the general case, indicated in the last remark in section 3. These results strongly indicate that there is a deeper link between the realm of (classically) integrable systems and the RG-flows. The last point especially demands for a deeper understanding of the Lie algebra of infinitesimal characters, respectively the Lie Group of regularized characters. We hope that this may be partly achieved by analyzing the link to the well established field of integrable systems.

\section{Acknowledgements}

The first author would like to thank the Ev. Studienwerk for financial support. Also the I.H.É.S. and its warm hospitality is greatly acknowledged.

\section{References}

[1] G. Baxter, An analytic problem whose solution follows from a simple algebraic identity, Pacific J. Math., 10, 731, (1960).

[2] F. V. Atkinson, Some aspects of Baxter's functional equation, J. Math. Anal. Appl., 7, 1, (1963).

[3] G.-C. Rota, Baxter algebras and combinatorial identities. I, II., Bull. Amer. Math. Soc. 75, 325, (1969); ibid. 75, 330, (1969).

[4] G.-C. Rota, Ten mathematics problems I will never solve, Mitt. Dtsch. Math.Ver., no. 2, 45, (1998).

[5] P. Cartier, On the structure of free Baxter algebras, Advances in Math., 9, 253, (1972). 
[6] G.-C. Rota, Baxter operators, an introduction, In: "Gian-Carlo Rota on Combinatorics, Introductory papers and commentaries", Joseph P.S. Kung, Editor, Birkhäuser, Boston, 1995.

[7] L. Guo, Baxter algebras and differential algebras, in "Differential algebra and related topics", (Newark, NJ, 2000), World Sci. Publishing, River Edge, NJ, 281, (2002).

[8] L. Guo, W. Keigher, Baxter algebras and shuffle products, Adv. Math., 150, no. $1,117,(2000)$.

[9] K. Ebrahimi-Fard, Loday-type algebras and the Rota-Baxter relation, Letters in Mathematical Physics, 61, no. 2, 139, (2002).

[10] Ph. Leroux, Ennea-algebras, Nov 2003, preprint: arXiv:math.QA/0309213.

[11] M. Aguiar, Prepoisson algebras, Letters in Mathematical Physics, 54, no. 4, 263, (2000).

[12] M. Aguiar, J.-L. Loday, Quadri-algebras, Sept. 2003, preprint: arXiv:math.QA/0309171, to appear in J. Pure Applied Algebra.

[13] A. Belavin, V. Drinfeld, Triangle Equations and Simple Lie-Algebras, Classic Reviews in Mathematics and Mathematical Physics, 1. Harwood Academic Publishers, Amsterdam, 1998, viii+91 pp.

[14] M. A. Semenov-Tian-Shansky, What is a classical r-matrix?, Funct. Ana. Appl., 17, no.4., 254, (1983).

[15] A. G. Reyman, M. A. Semenov-Tian-Shansky, Group theoretical methods in the theory of finite dimensional integrable systems, in: Encyclopedia of mathematical science, v.16: Dynamical Systems VII, Springer, 116, (1994).

[16] M. A. Semenov-Tian-Shansky, Integrable Systems and Factorization Problems, Lectures given at the Faro International Summer School on Factorization and Integrable Systems (Sept. 2000), Sept. 2002, preprint: arXiv: nlin.SI/0209057.

[17] D. Kreimer, On the Hopf algebra structure of perturbative quantum field theories, Adv. Theor. Math. Phys. 2, 303, (1998).

[18] D. Kreimer, Chen's iterated integral represents the operator product expansion Adv. Theor. Math. Phys., 3, no. 3, 627, (1999).

[19] A. Connes, D. Kreimer, Renormalization in quantum field theory and the Riemann-Hilbert problem. I. The Hopf algebra structure of graphs and the main theorem, Comm. in Math. Phys. 210, 249, (2000).

[20] A. Connes, D. Kreimer, Renormalization in quantum field theory and the Riemann-Hilbert problem. II. The $\beta$-function, diffeomorphisms and the renormalization group, Comm. in Math. Phys. 216, 215, (2001). 
[21] S. Weinzierl, Hopf algebra structures in particle physics, Talk given at International Europhysics Conference on High-Energy Physics (HEP 2003), Aachen, Germany, 17-23 Jul. 2003, preprint arXiv hep-th/0310124, (Oct. 2003).

[22] A. Connes, D. Kreimer, Hopf algebras, Renormalization and Noncommutative Geometry, Comm. in Math. Phys. 199, 203, (1998).

[23] H. Figueroa, J. M. Gracia-Bondia, J. C. Varilly, Elements of Noncommutative Geometry, Birkhäuser, (2001).

[24] D. Manchon, Hopf algebras, from basics to applications to renormalization, Comptes-rendus des Rencontres mathmatiques de Glanon 2001.

[25] A. Connes, D. Kreimer, Insertion and elimination: the doubly infinite Lie algebra of Feynman graphs, Ann. Henri Poincaré 3, no. 3, 411, (2002).

[26] I. Mencattini, D. Kreimer, Insertion and Elimination Lie Algebra: The Ladder Case, Lett. in Math. Phys., in print (2003) (math.QA/0308042).

[27] D. Kreimer, Factorization in quantum field theory: An exercise in Hopf algebras and local singularities, Proceedings From Number Theory to Physics and Geometry, Les Houches March 2003, in press, arXiv:hep-th/0306020.

[28] K. Ebrahimi-Fard, On the associative Nijenhuis relation, Feb 2003, preprint: arXiv:math-ph/0302062

[29] I.Z. Golubchik, V.V. Sokolov, One more type of classical Yang-Baxter equation, Funct. Anal. Appl., 34, no. 4, 296, (2000).

[30] Y. Kosmann-Schwarzbach, F.Magri, Poisson-Nijenhuis structures, Ann. Inst. H. Poincar Phys. Thor., 53, no. 1, 35, (1990).

[31] C. Chryssomalakos, H. Quevedo, M. Rosenbaum, J. D. Vergara, Normal Coordinates and Primitive Elements in the Hopf Algebra of Renormalization, Comm. in Math. Phys. 225, no. 3, 465, (2002).

[32] M. Sakakibara, On the Differential equations of the characters for the Renormalization group, Jan. 2004, preprint: arXiv:math-ph/0401048. 